\title{
Submicrometer Patterning of Charge in Thin-Film Electrets
}

\author{
Heiko O. Jacobs and George M. Whitesides
}

\begin{abstract}
Thin-film electrets have been patterned with trapped charge with submicrometer resolution using a flexible, electrically conductive electrode. A poly(dimethylsiloxane) stamp, patterned in bas-relief and supporting an 80-nanometer-thick gold film, is brought into contact with an 80-nanometer-thick film of poly(methylmethacrylate) supported on $n$-doped silicon. A voltage pulse between the gold film and the silicon transfers charge at the contact areas between the gold and the polymer electret. Areas as large as 1 square centimeter were patterned with trapped charges at a resolution better than 150 nanometers in less than 20 seconds. This process provides a new method for patterning; it suggests possible methods for high-density, charge-based data storage and for high-resolution charge-based printing.
\end{abstract}

Electrets are materials than can retain trapped electrical charge or polarization (1). Patterns of charge are used in photocopiers (xerography) to develop images with $100 \mu \mathrm{m}$ resolution (2). Systems that write and read patterns of charge have been explored extensively, because of their potential in rewritable digital data storage (3-5). Current procedures based on scanning probes achieve a writing rate of $100 \mathrm{kbits} / \mathrm{s}$ at an areal density of $7 \mathrm{Gbits} / \mathrm{cm}^{2}$ (120 nm bit size), and achieve a resolution of $100 \mathrm{~nm}(6,7)$. Although this density is about 140 times the areal density of optical compact discs, the writing rate is slow: patterning an area of $1 \mathrm{~cm}^{2}$ requires 24 hours. Here, we describe a method that uses a flexible, micropatterned electrode to pattern an electret thin film in a parallel process by injecting and trapping charges over areas of $\sim 1 \mathrm{~cm}^{2}$; we call this method electrical microcontact printing $(\mathrm{e}-\mu \mathrm{CP})$. Because the electrode is flexible, it can make sufficiently intimate electrical contact with a solid surface to produce uniform pattern transfer by charging. The resulting patterns were imaged using Kelvin probe force microscopy (KFM) (8). We have used e- $\mu \mathrm{CP}$ to pattern surfaces $\left(>1 \mathrm{~cm}^{2}\right)$ with features ranging from $120 \mathrm{~nm}$ to $100 \mu \mathrm{m}$ in size in less than $20 \mathrm{~s}$; this combination of area feature size, and writing time corresponds to an increase of $>10^{3}$ in writing speed compared to that obtained by a single tip in serial scanning probe methods.

Figure 1 illustrates the procedure. The stamp was poly(dimethylsiloxane) (PDMS), patterned in bas-relief using procedures described in (9); it was $\sim 5 \mathrm{~mm}$ thick and supported on a glass slide. The patterned surface of the PDMS stamp was made elec-

Department of Chemistry and Chemical Biology, Harvard University, 12 Oxford Street, Cambridge, MA 02138, USA.

E-mail: hjacobs@gmwgroup.harvard.edu (H.O.J.); gwhitesides@gmwgroup.harvard.edu (G.M.W.) trically conducting by thermal evaporation of $7 \mathrm{~nm}$ of $\mathrm{Cr}$ (as an adhesion promoter) and 80 $\mathrm{nm}$ of Au onto it (10). Poly(methylmethacrylate) (PMMA, an 80-nm film on a $<100>$ $n$-doped $\mathrm{Si}$ wafer with a resistivity of 3 $\mathrm{ohm} \cdot \mathrm{cm}$ ) was the charge storage medium; PMMA is commercially available and is an electret with good charge storage capabilities (11). The wafer was cut into $1-\mathrm{cm}^{2}$ squares. To generate a pattern of trapped charge, we placed the metal-coated PDMS stamp on top of the PMMA film (without added pressure) and applied a voltage of 10 to $20 \mathrm{~V}$ between the Au on the PDMS and the back side of the Si. During the patterning, we monitored the current (typically 1 to $20 \mathrm{~mA} / \mathrm{cm}^{2}$ ) and the total charge transferred through the entire junction (10 to $100 \mathrm{mC} / \mathrm{cm}^{2}, 600$ to 6000 electrons $/ \mathrm{nm}^{2}$ ). After turning off the applied potential, we removed the PDMS stamp by hand.

Several representative patterns of localized charge in PMMA generated by e- $\mu \mathrm{CP}$ were recorded by KFM (Fig. 2). The measured topography (Fig. 2A) and the distribution of the surface potential (Fig. 2B; light $=$ positive potential) are shown for a surface patterned in a way that simulates high-density data storage [full width at half-maximum $(\mathrm{FWHM})<150 \mathrm{~nm}$, density $\left.=5 \mathrm{Gbits} / \mathrm{cm}^{2}\right]$. To write this charge pattern, we exposed the PMMA film locally with a current density of $20 \mathrm{~mA} / \mathrm{cm}^{2}(18 \mathrm{~V})$ for $10 \mathrm{~s}$ with the metalcoated stamp having positive charge. The trapped charges change the local potential at the surface of the PMMA by about $\sim 1 \mathrm{~V}$, whereas the topography of the surface of the PMMA is not significantly changed. Figure 2, C and D, shows two areas of a common surface that were charged in a single step with patterns of different dimensions. The stamps can be used many times: the $10-\mu \mathrm{m}-$ wide charged lines in Fig. 2E were patterned with a stamp that had been used 14 times previously. These patterns are representative of those observed over large areas $\left(>1 \mathrm{~cm}^{2}\right)$. Figure 2F shows an image of negative charge in PMMA formed by a $-20 \mathrm{~V}$ voltage pulse of $15 \mathrm{~s}$ duration. The smallest charged areas we have generated are about $150 \mathrm{~nm}$ wide. At these scales, the transfer function of the Kelvin probe limits the resolution (12).

To investigate the temporal stability of the charge, we compared the charge pattern imaged immediately after formation (Fig. 3A) with the charge pattern of the same sample
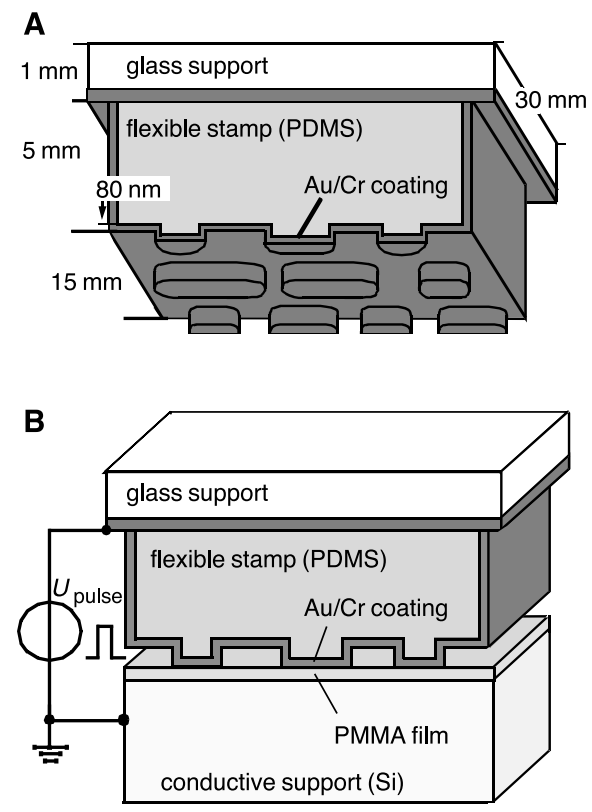

C

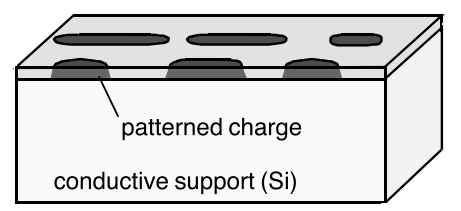

Fig. 1. Principle of electrical microcontact printing $(\mathrm{e}-\mu \mathrm{CP})$. (A) The flexible, metal-coated stamp is placed on top of a thin film of PMMA supported on a doped, electrically conducting Si wafer. We used a $2 \%$ solution of $950 \mathrm{~K}$ PMMA in chlorobenzene (MicroChem, Newton, Massachusetts) and spin-coating at $6000 \mathrm{rpm}$ to form the film on the wafer. The film was baked at $90^{\circ} \mathrm{C}$ for 1 hour under vacuum. (B) An external voltage was applied between the $\mathrm{Au}$ and the Si to write the pattern of the stamp into the electret. Electrical connections to the Au-coated PDMS stamp and the $n$-doped $\mathrm{Si}$ were applied using silver paint (Aldrich) and InGa (a liquid metal alloy, Aldrich), respectively. Silver paint was applied to the interface between the glass support and the stamp to make a good electrical contact between both surfaces. Silver paint was also used to connect the glass substrate to the power supply with a flexible $\mathrm{Cu}$ wire. InGa was spread over the back side of the Si chips. The chips were then placed on a Cu plate that was connected to the power supply via an electrometer. (C) The stamp was removed; the PMMA was left with a patterned electrostatic potential. 


\section{RE P O R T S}

aged in air (Fig. 3B) and exposed to deionized water (Fig. 3C). In air, at room temperature, the amplitude of the potential decreased from 1.8 to $1.6 \mathrm{~V}$ over an interval of 2 months. Between measurements, the PMMA was kept in a covered petri dish at ambient conditions. The stability we observed was larger than that reported for thick $(>100 \mu \mathrm{m})$ PMMA films charged by corona discharge (11). We do not understand the difference, but note that the mechanisms of charging and the depth of charge injection into the polymers are plausibly different and noncomparable in these two procedures. In deionized water, the potential decreased from 1.8 to $0.3 \mathrm{~V}$ within $30 \mathrm{~min}$ (13). The patterns appear not to diffuse laterally either on standing in air or on exposure to water. A positively charged pattern could be discharged almost completely by applying negative ions (primarily $\mathrm{CO}_{3}{ }^{-}$) from a corona discharge using an electrostatic gun (Fig. 3D) (14) or by heating the PMMA to above its glass transition temperature (15).

The charging of the polymer electrets in these experiments could, in principle, be explained by two different types of mechanisms: (i) transfer of electrons (or, perhaps, ions) from the electrodes to or from the film or (ii) orientation of dipoles inside the film by
Fig. 2. KFM images of patterns of positive and negative surface potential generated by e- $\mu$ CP. (A) Surface topography and (B) surface potential distribution of a test pattern of high-density data storage with $<150-\mathrm{nm}$ sized bits (FWHM). The pattern was generated using a stamp carrying 150-nm-wide circular posts that were $90 \mathrm{~nm}$ high. (C and D) Surface potential images of ring-type charge patterns generated using a stamp carrying 350- and 640-nm-wide circular posts that were slightly higher at the edge than in the center. (E) Surface potential image of positively charged parallel lines generated using a stamp carrying 10 - $\mu \mathrm{m}$-wide parallel lines that were $850 \mathrm{~nm}$ high. (F) Surface potential images of negatively charged dots generated using a stamp carrying $2.5-\mu \mathrm{m}$-wide circular posts that were $850 \mathrm{~nm}$ high. The amplitudes of the surface potentials in these figures cannot be compared directly. the applied electric field (16). When the stamp was positively charged, the resulting surface potential was positive (Fig. 3). This polarity cannot be explained solely on the basis of dipole orientation: Dipoles would orient with their negative end toward the surface in contact with a positively charged electrode. We therefore infer that the mechanism of charging is dominated by the transport of electrons (or in principle, although less probably, ions) into and/or out of the PMMA film.

Direct charge transfer between the Au and the PMMA can only be explained if the stamp contacts the electret. To explore the ability of the flexible PDMS stamp to form a spatially close contact to a rigid surface, we compared two systems of patterned electrodes: (i) a rigid Si wafer supporting a pattern in metal $(7 \mathrm{~nm}$ of $\mathrm{Cr}$ and $80 \mathrm{~nm}$ of $\mathrm{Au}$, thermally evaporated) and (ii) a flexible, metal-coated PDMS stamp. Figure 4 shows two optical micrographs of the optical interference pattern observed at the interface between the PMMA film and these rigid and flexible electrodes. The rigid pattern transfer element always displayed optical interference patterns; these patterns indicate a nonuniform spacing between the substrate and the patterning element. At best, by using significant pressure, we could obtain small $(<500 \mu \mathrm{m}$ in diameter) areas that showed no optical interference phenomena and that seemed to be in contact. The electric contact resistance of such contacts, however, depended strongly on the applied pressure, the rigidity of both surfaces, and the amount and type of contamination at the interface. We were unsuccessful in using a rigid plate of this type for patterning.

Replacing the rigid electrode with the elastomeric PDMS/Au electrode produced large areas showing no optical interference patterns and which were apparently in contact with the substrate. Figure 4C shows a scanning electron microscope (SEM) image of the interface (obtained by placing a rigid Si substrate on top of a flexible stamp, see inset). We observed no spacing (we would be able to observe gaps $>10 \mathrm{~nm}$ ) between the Si substrate and the stamp. This observation provides evidence that intimate contact between electrode and substrate can be established with a rigid surface. We do not know if contacts that are intimate in this sense are necessarily in van der Waals contact over the majority of their proximate area. The Au film
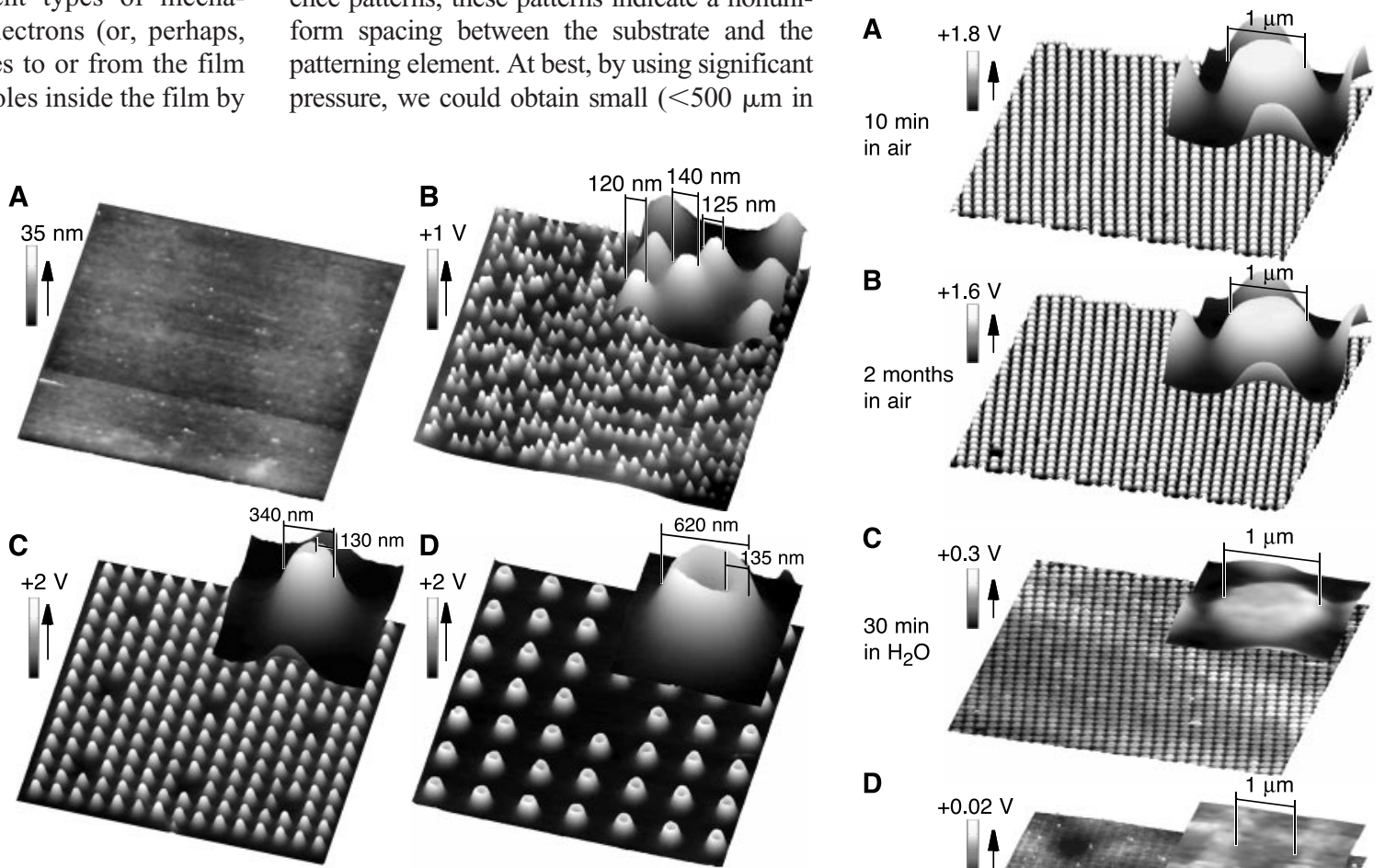

C

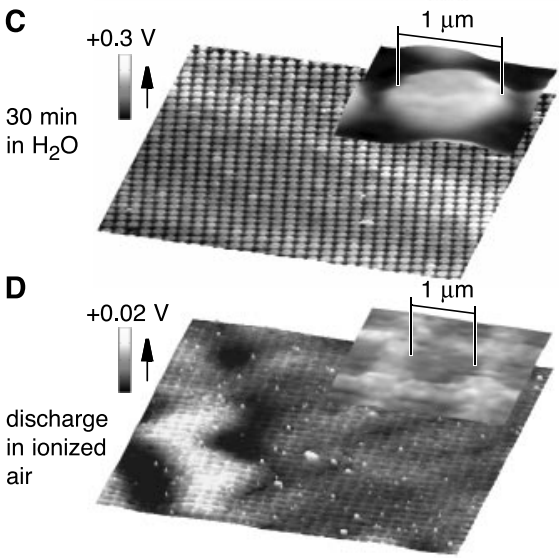

Fig. 3. Stability of the patterned charge in air, water, and ionized air. Surface potential distribution recorded (A) immediately after formation and (B) after 2 months aging in air. (C) Surface potential distribution upon exposing the charge pattern to deionized water. (D) Surface potential distribution upon discharge with ionized air. 


\section{R E P O R T S}

of the PDMS stamp has a surface roughness of about $5 \mathrm{~nm}$. In addition to relatively large deformations of the PDMS stamp due to pressure and van der Waals forces, the electrical potential applied between the stamp and the Si supporting the PMMA film could also promote contact between the $\mathrm{Au}$ and the PMMA. For flat and clean surfaces, no external pressure was required to obtain contact sufficient to give uniform electrical patterning at $\sim 150-\mathrm{nm}$ dimensions (17).

In principle, local heating could also cause the PMMA to deform plastically. Melting is obvious in the 80 -nm-thick PMMA film after $10 \mathrm{~s}$ at a current density $>100$ $\mathrm{mA} / \mathrm{cm}^{2}$. At these current densities, the stamp sinks into the PMMA and leaves a negative topographic image on the surface. Because we could pattern charge with current densities $<1 \mathrm{~mA} / \mathrm{cm}^{2}$ - a thermal energy $10^{4}$ smaller than what is needed to melt the PMMA - we conclude that bulk, heat-induced melting and flow are not necessary for patterning, but we cannot preclude surface flow on scales of a few nanometers as a result of current-induced warming and electrostatic pressure.

We believe that the ability to generate high-resolution patterns of trapped charge can, in principle, be used (i) for information storage, (ii) for second-harmonic generation of optical waves traveling along a surface (18-21), (iii) to deplete and pattern regions of a two-dimensional electron gas $(22,23)$ near a semiconductor surface, and (iv) to allow the realization of a charge-based printing technique analogous to xerography of small particles and possibly molecules (24). Figure 5 shows an initial example of charge-based printing of particles. The images show patterns of carbon toner, iron beads, and red iron oxide that were trapped at charged areas on PMMA and subsequently transferred onto a second substrate. In this procedure, we dipped PMMA-coated chips carrying a positively charged pattern into dry powders (25) and developed the pattern by blowing away the loosely held material in a stream of dry nitrogen. To transfer the powder pattern onto a second substrate, we placed the powdercoated face of the chips on a flat surface of polymerized PDMS (Fig. 5, A and C) or on an adhesive surface of Scotch tape (Fig. 5B), pressed down slightly on the chips to make contact, and peeled away the flexible films. The resolution achieved was $50 \mu \mathrm{m}$ for $\mathrm{Xe}$ rox toner and $2 \mu \mathrm{m}$ for red iron oxide.

Electrical microcontact printing extends previous serial techniques for patterning charge $(2,3)$ into a parallel method, and provides the only parallel method now available for patterning charge in electrets with resolution greater than $150 \mathrm{~nm}$. The areal uniformity, resolution, and stability of the these patterns are remarkable. The observed electrostatic potential corresponds to $\sim 100$ elementary charges per $10^{4} \mathrm{~nm}^{2}$ of surface (26). These patterns form as a result of the passage of substantial currents through the films, but we have not established the atomic/ molecular-level details of either the contact between the surfaces of the electrodes and the polymer films or of the processes that ultimately generate the trapped charges and fixed dipoles. We also have not established the minimum current density and exposure time that is required for patterning.

This method for patterning charge is interesting both as a tool for fundamental studies of electrets and for its potential in applications. It will allow studies of the materials science of thin-film electrets at high lateral resolution. It also suggests routes to highdensity ( $\left.>5 \mathrm{Gbits} / \mathrm{cm}^{2}\right)$ data storage; to ma-
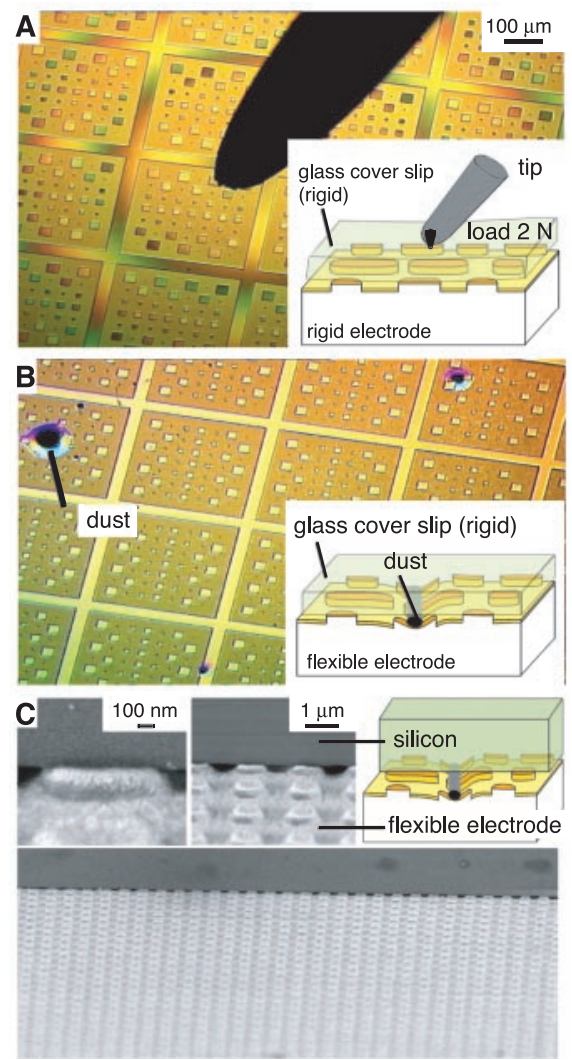

Fig. 4. Optical and SEM micrographs of contact between two rigid surfaces $[(A)$ glass cover slip and $\mathrm{Au}$-coated $\mathrm{Si}$ and rigid/flexible surfaces [(B) glass cover slip and Au-coated PDMS and (C) Si- and Au-coated PDMS]. (A) Top view of the optical interference pattern observed at the interface of the rigid/rigid contact; This pattern indicates separations on the order of the optical wavelength. Spacings comparable to optical wavelength are obtained only at high contact pressures (see inset). (B) The rigid/flexible contact. The flexible master electrode deforms around dust particles (see inset) and forms intermediate electric microcontacts. No interference is visible. (C) SEM images of a rigid/ flexible contact, which shows that the spacing is well below $20 \mathrm{~nm}$. terials with patterned dipoles, indices of refraction, and color centers; and to micrometer-scale electrostatic printing techniques resembling xerography. It may also be possible to extend this method to increase the rate of other processes (especially serial processes such as scanning probe lithography and ebeam writing) that use electrons to write patterns: formation of oxides on surfaces (27) and exposure of electron-sensitive resists (28) are attractive candidates.
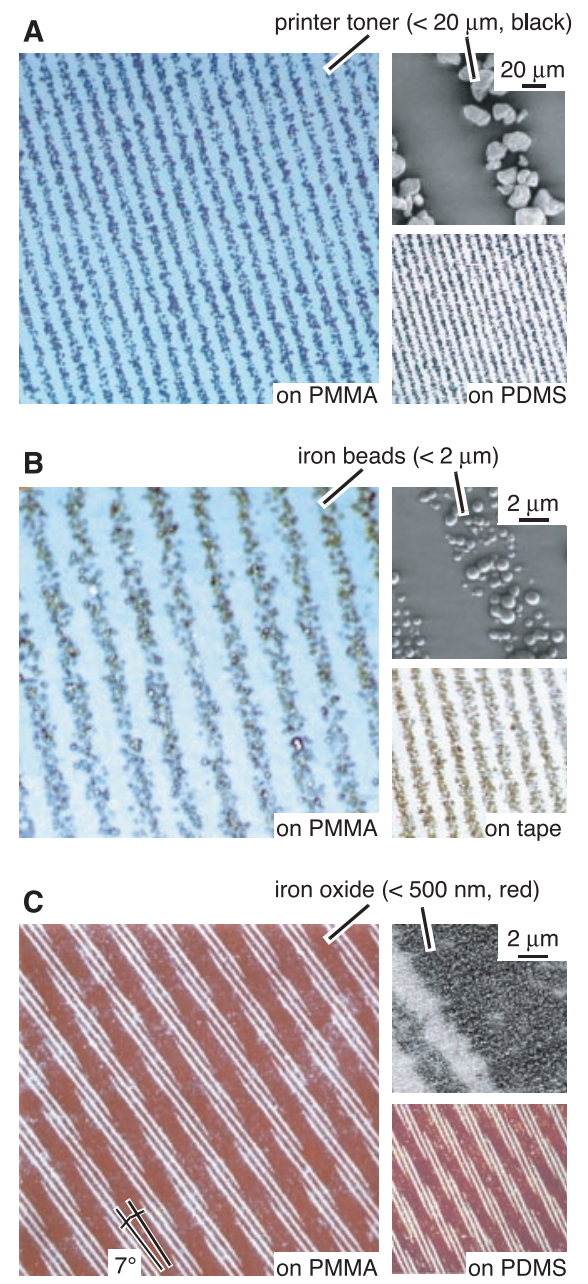

Fig. 5. Optical microscope and SEM images of different types of particles trapped at patterned charge and transferred onto second substrates. (A) $50-\mu \mathrm{m}$-wide parallel lines of toner particles, $<20 \mu \mathrm{m}$ in size; (B) $5-\mu \mathrm{m}$-wide parallel lines of iron beads, $<2 \mu \mathrm{m}$ in size; (C) 2.5and $10-\mu \mathrm{m}$-wide lines of red iron oxide particles, $<500 \mathrm{~nm}$ in size, crossing at $7^{\circ}$ incidence. In this demonstration, all particles were trapped with positive charge that was patterned with stamps carrying parallel lines $2.5,5$, and $10 \mu \mathrm{m}$ wide. The crossing lines were fabricated by two exposures at different angles. The insets labeled "on tape" and "on PDMS" are images of patterns of particles transferred to Scotch tape and PDMS, respectively, by contact. All optical images are in true color. Contrast and brightness have been adjusted slightly. 
References and Notes

1. G. M. Sessler, Electrets (Springer-Verlag, Berlin, 1987)

2. D. M. Pai, B. E. Springett, Rev. Mod. Phys. 65, 163 (1993).

3. R. C. Barrett, C. F. Quate, J. Appl. Phys. 70, 2725 (1991).

4. M. Dreyer, R. Wiesendanger, Appl. Phys. A 61, 357 (1995).

5. H. O. Jacobs, A. Stemmer, Surf. Interface Anal. 27, 361 (1999).

6. H. J. Mamin et al., IBM J. Res. Dev. 39, 681 (1995).

7. A. Born, R. Wiesendanger, Appl. Phys. A 68, 131 (1999).

8. H. O. Jacobs, H. F. Knapp, S. Müller, A. Stemmer, Ultramicroscopy 69, 39 (1997)

9. Y. Xia, G. M. Whitesides, Angew. Chem. Int. Ed. Engl. 37, 550 (1998).

10. Thermal expansion of the PDMS stamps during the evaporation can cause the metal coating to buckle on cooling [N. Bowden, S. Brittain, A. G. Evans, J. W. Hutchinson, G. M. Whitesides, Nature 393, 146 (1998)]. This buckling can be prevented by mounting the PDMS stamps at a distance from the metal source that is sufficient to limit thermal heating. In the resistive thermal evaporator (Cryo Auto 306, Edwards High Vacuum International, Wilmington, MA), a distance of $25 \mathrm{~cm}$ was sufficient to obtain a flat $\mathrm{Au}$ coating on the PDMS stamps.

11. H. S. Nalwa, Ferroelectric Polymers: Chemistry, Physics and Applications (Dekker, New York, 1995); K. Mazur, J. Phys. D 30, 1383 (1997).

12. The resolution of the Kelvin probe is limited because of the finite dimensions of the probes. For the probes we used in the experiment (Nanosensors, Dr. O. Wolter GmbH, Wetzlar-Blankenfeld, Germany), a lateral potential step on the surface is detected as a smoothed curve about $80 \mathrm{~nm}$ wide [H. O. Jacobs, P. Leuchtmann, O. J. Homan, A. Stemmer, J. Appl. Phys. 84, 1168 (1998)] and small $(<100 \mathrm{~nm})$ charged areas appear broader and more diffuse in the recorded images than they actually are (Fig. 2).

13. The charged PMMA surface was covered with a drop (30 $\mu \mathrm{l})$ of deionized water (resistance: 10 megohms) for $3 \mathrm{~min}$ for the first experiment (not shown), followed by another $30 \mathrm{~min}$ for the second experiment. Before characterizing the charge patterns, we dried the surface of the PMMA under a stream of dry nitrogen.

14. The corona discharge occurs at the front of the electrostatic gun (Zerostat, Aldrich) between two electrodes. A slow release of the trigger, lasting about $2 \mathrm{~s}$, emits a stream of negatively ionized air \{mainly $\mathrm{CO}_{3}{ }^{-}[(1)$, p. 30]\}. The positively charged PMMA was neutralized by releasing the handle 10 times, with the gun placed $1 \mathrm{~cm}$ above the surface.

15. The chip was heated for $10 \mathrm{~min}$ at $130^{\circ} \mathrm{C}$ on a hot-plate at ambient pressure and humidity. The glass transition temperature of PMMA is $105^{\circ} \mathrm{C}$ [D. R. Lide, Handbook of Chemistry and Physics (CRC, Boca Raton, FL, ed. 80, 2000), p. 13-6].

16. J. D. Jackson, Classical Electrodynamics (Wiley, New York, 1975)

17. When the surfaces are contaminated with dust particles, and the weight of the stamp provides insufficient pressure to deform around these particles, external pressure can become necessary to establish contact.

18. Oriented dipoles and trapped charges in electrets have be used to generate shorter wavelength light from a longer wavelength source [see (19-21), and references therein].

19. M. A. Pauley et al., J. Chem. Phys. 104, 6834 (1996).

20. S. Bauer-Gogonea, R. Gerhard-Multhaupt, IEEE Trans. Dielectr. Electr. Insul. 3, 677 (1996).

21. J. Fang, G. P. Li, Appl. Phys. Lett. 75, 3506 (1999).

22. U. F. Keyser et al., Appl. Phys. Lett. 76, 457 (2000).

23. M. A. Topinka et al., Science 289, 2323 (2000).

24. W. M. D. Wright, D. G. Chetwynd, Nanotechnology 9, 133 (1998).

25. The black toner (product number 13R55) was obtained from Xerox Co., and the red iron oxide and iron beads was obtained from PolyScience (Niles, IL).

26. We assume that trapped charge inside or on the surface of the PMMA film will attract mobile charge carriers inside the Si substrate. This displacement of charge carriers will result in the formation of a double layer. For a double layer separated by a distinct distance $d$, the charge density $\sigma$ can be calculated with $\sigma=\varepsilon \Delta V / d$, where $\varepsilon$ is the permittivity, and $\Delta V$ is the voltage drop across the layer (16). For $\varepsilon=8 \times 10^{-12} \mathrm{C} / \mathrm{Vm}$ (permittivity of PMMA), $\Delta V=2 \mathrm{~V}$ (measured potential change), and $d=100 \mathrm{~nm}$ (assumed intermediate distance between the counter charges), we obtain a firstorder estimate of the effective charge density of $\sigma_{\text {eff }}=$ 100 elementary charges per surface area of $100^{2} \mathrm{~nm}^{2}$ The exact number depends on the actual distribution of the charges inside the PMMA film and the Si substrate; we presently have no information on this distribution.

27. B. Legrand, D. Stievenard, Appl. Phys. Lett. 74, 4049 (1999)
28. K. Wilder, D. Adderton, R. Bernstein, V. Elings, C. F. Quate, Appl. Phys. Lett. 73, 2527 (1998).

29. We thank Th. Schimmel, A. Stroock, A. Stemmer, and A. Schwartz for helpful discussions; J. Ng, P. Deschatelets, and J. Wiles for suggestions on charge-based printing; K. Paul for help on stamp fabrication; and $\mathrm{M}$. Tinkham for allowing us to use his atomic force microscope. This work was supported by the Swiss National Science Foundation (SNSF), by the Deutsche Forschungsgemeinschaft, and by Defense Advanced Research Projects Agency/AFRL/SPAWAR.

31 October 2000; accepted 24 January 2001

\title{
Fluorous Mixture Synthesis: A Fluorous-Tagging Strategy for the Synthesis and Separation of Mixtures of Organic Compounds
}

\author{
Zhiyong Luo, ${ }^{1,2}$ Qisheng Zhang, ${ }^{1}$ Yoji Oderaotoshi, ${ }^{1}$ \\ Dennis P. Curran ${ }^{1 *}$
}

The solution-phase synthesis of organic compounds as mixtures rather than in individual pure form offers efficiency advantages that are negated by the difficulty in separating and identifying the components of the final mixture. Here, a strategy for mixture synthesis that addresses these separation and identification problems is presented. A series of organic substrates was tagged with a series of fluorous tags of increasing fluorine content. The compounds were then mixed, and multistep reactions were conducted to make enantiomers or analogs of the natural product mappicine. The resulting tagged products were then demixed by fluorous chromatography (eluting in order of increasing fluorine content) to provide the individual pure components of the mixture, which were detagged to release the final products.

We introduce a technique for the synthesis of mixtures of organic compounds that simultaneously solves both the separation and identification problems heretofore inherent in solution-phase mixture synthesis. The technique, called fluorous mixture synthesis (FMS), follows from early fluorous techniques such as fluorous biphasic catalysis (1-3) and solution-phase synthesis of small organic molecules by fluorous tagging $(4,5)$ (sometimes called "fluorous synthesis"). We envision a broad range of applications for FMS techniques, and here we introduce two applications: quasiracemic synthesis and parallel library synthesis. Both are illustrated in the context of the natural product mappicine $(6,7)$.

The synthesis of small organic molecules can be conducted either in solution or on the solid phase $(8)$. When a single product or a

'Department of Chemistry and Center for Combinatorial Chemistry, University of Pittsburgh, Pittsburgh, PA 15260, USA. ${ }^{2}$ Fluorous Technologies, U-PARC, 970 William Pitt Way, Pittsburgh, PA 15238, USA.

*To whom correspondence should be addressed. few products are targeted, solution-phase synthesis is almost always used. When larger numbers of products are targeted, the options include solution-phase parallel synthesis, solid-phase parallel synthesis, and solid-phase mixture (split-mix) synthesis $(9-11)$. Solution-phase synthesis allows for diverse and homogeneous reaction conditions, and products are readily analyzed and identified, but purifications are time-consuming and keeping all samples spatially separate is inefficient. Solid-phase synthesis renders separation of excess or spent reagents and reactants easy, and the efficiency of mixture methods allows more compounds to be made without a proportional increase in effort. But the scope of reactions that succeed on the solid phase is still limited, as are methods for purification, analysis, and identification of resin-bound products.

Ideal solution-phase synthesis methods would retain the reaction, identification, and analysis features of traditional solution-phase synthesis while capturing the separation and mixture advantages of solid-phase synthesis. Although there has been much work recently 